\title{
Linear-quadratic programming and its application to data correction of improper linear programming problems
}

https://doi.org/10.1515/comp-2020-0005

Received Sep 01, 2019; accepted Dec 05, 2019

\begin{abstract}
The problem of maximizing a linear function with linear and quadratic constraints is considered. The solution of the problem is obtained in a constructive form using the Lagrange function and the optimality conditions. Many optimization problems can be reduced to the problem of this type. In this paper, as an application, we consider an improper linear programming problem formalized in the form of maximization of the initial linear criterion with a restriction to the Euclidean norm of the correction vector of the right-hand side of the constraints or the Frobenius norm of the correction matrix of both sides of the constraints.
\end{abstract}

Keywords: linear programming, quadratic programming, improper problem, data correction

\section{Introduction}

The problem of quadratic programming is the problem of maximizing a quadratic function (possibly with a linear part) with quadratic and/or linear constraints. In general, this class of problems is computationally difficult. For some subclasses of quadratic programming problems, in particular convex problems and semi-definite programming problems [1], polynomial complexity is proved, however, the corresponding algorithms (for example, the cut method, the ellipsoid method) have rather slow convergence. Therefore, it is interesting to find out more simple classes which contain a number of interesting applied problems and which solutions partly in the analytical form can be obtained. In this connection, we'll consider the problem of maximizing a linear function with

\footnotetext{
*Corresponding Author: Victor Gorelik: Dorodnicyn Computing Centre FRC CSC RAS, Moscow, Russia; Email: vgor16@mail.ru Tatiana Zolotova: Financial University under the Government of RF, Moscow, Russia; Email: tgold11@mail.ru
}

one quadratic and several linear constraints and call it the problem of linear-quadratic programming. Many optimization problems can be reduced to it. Important examples of such problems are stochastic, improper and ill-posed problems of linear programming (LP). So the linear problem of stochastic programming is usually formalized in the form of maximizing the mathematical expectation of the objective function under the condition that the probability of the constraints fulfilling is not less than a given value, and under the assumption of a normal distribution of the initial parameters can be reduced to a linear-quadratic problem.

Now data correction (approximation) methods for improper and unstable problems are widely used. Vector correction (right side) and matrix correction (of all initial data) are applied to inconsistent systems of linear algebraic equations and inequalities and improper problems of LP (regression analysis [2,3], production planning [4, 5], etc.). If the obtained correction problem of LP has no solution, then the correction of a pair of dual problems may be considered [6]. However, there is another problem. The optimal correction in the form of minimizing the Euclidean norm of the right-hand side vector or the Frobenius norm of the correction matrix, as a rule, has a unique solution and the maximization of the initial criterion does not make sense. Therefore, if for inconsistent systems of equalities and inequalities the problem of minimal correction is quite natural, then for improper optimization problems it does not solve completely the question. In fact, here we are dealing with a two-criteria problem: maximizing the original criterion and minimizing the norm of the correction matrix.

So, in [5], the formalization of the improper LP problem with inconsistent constraints was proposed as the problem of minimizing the spectral or Frobenius norm of the data correction matrix with a lower bound on the initial criterion and its solution was found. This yields the same class of optimization problems as for the minimal correction, namely, the original problem with bilinear constraints can be reduced using the Euclidean norm to the This work is licensed under the Creative Commons

๑ Open Access. (C) 2020 V. Gorelik and T. Zolotova, published by De Gruyter. Attribution 4.0 License 
classical quadratic programming problem (a quadratic objective function and linear constraints), and using polyhedral norms - to LP problems. Such an approach can be applied to the proper LP problems, in which the optimal value of the criterion on the initial admissible set is unsatisfactory from the point of view of the decision-maker.

We propose a new approach to the two-criteria problem of improper LP problems correction. This approach consists in formalizing these problems in the form of maximization of the initial criterion with a restriction to the spectral norm or the Frobenius norm of the correction matrix. Then we obtain problems of linear-quadratic programming. Also, if the improper problem of quadratic programming is formalized in the form of minimizing the $l_{1}$-norm of the data correction matrix (for linear constraints) with restriction to the initial criterion value, we obtain a similar problem. To the best of the authors' knowledge, this approach to the formulation of the improper problems correction was not considered.

In [7] the solution of the linear-quadratic programming problem was found in the explicit form and as an application of the results obtained, two problems of the optimal investment portfolio selection were considered. The first relates to the solution of the problem of maximizing the expected return with a limitation on the variance, the second - with a limitation on the probability of negative returns.

In order to make the exposition more self-contained and for the convenience of the reader, next we reproduce the result of [7: section 2]. In this paper we prove the theorem of a solution existence of the linear-quadratic programming problem, which clarifies this result. But the main content of the work is to present new results concerning its application to the solution of the task of the improper LP problem data correction with a restriction to the Euclidean norm of the correction vector of the right-hand side or the Frobenius norm of the correction matrix of both sides of the constraints. Within the framework of this approach, the problems of maximizing a linear function with a quadratic restriction arise. Note that in [8] we have considered the task of the improper LP problem data correction with a restriction to the Frobenius norm of the correction matrix of the left-hand side of the constraints, which has certain specificity and demanded a slightly different approach.

The improper LP problems considered below are presented in canonical form, i.e. with constraints of the equality type. Therefore, the correction problems are reduced to maximizing a linear function with one quadratic constraint of the inequality type and an arbitrary number of linear equality constraints.
In all problems we consider two cases: (a) the absence of the condition of non-negativity of variables, (b) the presence of this condition. Generally speaking, the case (a) has an independent practical meaning (for example, in [7], the tasks of stock investment in the presence of short sales were considered as an application, variables of arbitrary sign may be present in balance and flow models, etc.). In this case, the improper LP problems are associated with the contradictory nature of the constraints (mismatch of capacities and needs). Then, as a rule, we deal with the over-determined system of equations, which is often encountered in modeling and is the main assumption in this work. In case (b), the improper problem may be related either with the absence of a non-negative solution of an under-determined system of equations, or again with an over-determined system. We confine ourselves to the latter (the first sub-case generates other mathematical problems of projection).

\section{Maximization of a linear function with linear and quadratic constraints}

The mathematical formulation of the linear-quadratic programming problem has the form

$$
\langle c, x\rangle \rightarrow \max _{x \in X}, X=\{x \mid\langle D x, x\rangle \leq d, A x=b\} .
$$

Here $x \in E^{n}, c \in E^{n}, b \in E^{m}$ are column vectors, $\langle\cdot, \cdot\rangle$ denotes the scalar product of vectors, $D$ is a symmetric positive definite matrix of a size $n \times n, A$ is a matrix of a size $m \times n$, $m<n$. The superscript $T$ denotes further the transpose of a vector or a matrix.

Theorem 1. If $d\rangle\left\langle\left(A D^{-1} A^{T}\right)^{-1} b, b\right\rangle, \operatorname{rank} A=m$, the vector $c$ does not belong to the subspace of vector-rows of the matrix $A$, the system of equations $A x=b$ is consistent, then the problem (1) has a solution and it can be represented in the form

$$
\begin{aligned}
x^{0} & =\frac{1}{2 \lambda^{0}} D^{-1}\left(c-A^{T} \mu^{0}\right), \\
\lambda^{0} & =\frac{1}{2} \sqrt{\frac{\left\langle D^{-1} c, c-A^{T}\left(A D^{-1} A^{T}\right)^{-1}\left(A D^{-1} c\right)\right\rangle}{d-\left\langle\left(A D^{-1} A^{T}\right)^{-1} b, b\right\rangle},} \\
\mu^{0} & =\left(A D^{-1} A^{T}\right)^{-1}\left(A D^{-1} c-2 \lambda^{0} b\right) .
\end{aligned}
$$

Proof. The set $X$ is not empty if the system $A x=b$ is consistent and $d \geq d_{0}$, where

$$
d_{0}=\min _{x \in X_{0}}\langle D x, x\rangle, X_{0}=\{x \mid A x=b\} .
$$


Let's find $d_{0}$, solving the auxiliary problem of quadratic programming (3). We compose the Lagrange function: $L_{0}(x, \mu)=\langle D x, x\rangle+\langle\mu, b-A x\rangle$, differentiate it with respect to $x$ and equate to zero the partial derivatives: $2 D x-A^{T} \mu=0$. Since the matrix $D$ is nondegenerate, we have $x=\frac{1}{2} D^{-1} A^{T} \mu$ and from the constraint in (3) $A D^{-1} A^{T} \mu=2 b$. Let's introduce the denotation $R=A D^{-1} A^{T}$. The matrix $R$ is symmetric and positive definite, so $R$ is non-degenerate. Then we obtain $\mu=2 R^{-1} b$ and $x=D^{-1} A^{T} R^{-1} b$. Substituting $x$ in the function (3), we have $d_{0}=\left\langle A^{T} R^{-1} b, D^{-1} A^{T} R^{-1} b\right\rangle=$ $\left\langle R^{-1} b, A D^{-1} A^{T} R^{-1} b\right\rangle=\left\langle R^{-1} b, b\right\rangle$.

Let's return to the main problem (1). If $d=d_{0}$, then the set $X$ consists of one point $x=D^{-1} A^{T} R^{-1} b$ and the problem (1) becomes trivial. So we have assumed, that $d>d_{0}$. Obviously, the set $X$ is bounded, therefore the problem (1) has a solution. For this problem the Lagrange function has the form:

$$
L(x, \lambda, \mu)=\langle c, x\rangle+\lambda(d-\langle D x, x\rangle)+\langle\mu, b-A x\rangle,
$$

where $\lambda \geq 0$. The problem (1) is a convex programming problem that satisfies the Slater condition under the assumptions made. Therefore, by Karush-Kuhn-Tucker (KKT) theorem (known also as Kuhn-Tucker theorem), in order that $x^{0}$ be a solution of (1), it is necessary and sufficient that there exists such $y^{0}=\left(\lambda^{0}, \mu^{0}\right)$ that the pair $\left(x^{0}, y^{0}\right)$ is a saddle point of the Lagrange function $L$. To find the saddle point $\left(x^{0}, y^{0}\right)$, we differentiate the function $L$ with respect to $x$ and equate the derivatives to zero: $c-2 \lambda D x-A^{T} \mu=0$. In this equation $\lambda \neq 0$ because otherwise $c=A^{T} \mu$, which contradicts the assumption that the vector $c$ does not belong to the subspace of vector-rows of $A$. Therefore, the constraint $\langle D x, x\rangle \leq d$ is essential, i.e. at the solution point it is active, and $\lambda>0$. Then $x=\frac{1}{2 \lambda} D^{-1}\left(c-A^{T} \mu\right)$. We substitute $x$ into the constraints of the problem (1):

$$
\begin{aligned}
A x & =\frac{1}{2 \lambda} A D^{-1}\left(c-A^{T} \mu\right)=b, \\
\langle D x, x\rangle & =\frac{1}{2 \lambda}(\langle c, x\rangle-\langle\mu, A x\rangle) \\
& =\frac{1}{4 \lambda^{2}}\left\langle c, D^{-1}\left(c-A^{T} \mu\right)\right\rangle-\frac{1}{2 \lambda}\langle\mu, b\rangle=d .
\end{aligned}
$$

We denote by $r=A D^{-1} c$. From the first equality we have $r-R \mu=2 \lambda b$, whence $\mu=R^{-1}(r-2 \lambda b)$. We substitute $\mu$ into the second equality

$$
\begin{aligned}
& \left\langle D^{-1} c, c\right\rangle-\left\langle c, D^{-1} A^{T} R^{-1}(r-2 \lambda b)\right\rangle \\
& -2 \lambda\left\langle R^{-1}(r-2 \lambda b), b\right\rangle=4 \lambda^{2} d
\end{aligned}
$$

and obtain the next quadratic equation with respect to $\lambda$ :

$$
4\left(d-\left\langle R^{-1} b, b\right\rangle\right) \lambda^{2}+2\left(\left\langle R^{-1} r, b\right\rangle\right.
$$

$$
\begin{aligned}
& \left.-\left\langle c, D^{-1} A^{T} R^{-1} b\right\rangle\right) \lambda-\left\langle D^{-1} c, c\right\rangle \\
& +\left\langle c, D^{-1} A^{T} R^{-1} r\right\rangle=0 .
\end{aligned}
$$

By virtue of the transpose properties of the matrix product and the symmetry of the matrices $R^{-1}$ and $D^{-1}$ the coefficient at $\lambda$ in equation (4) is zero, and the free term is negative. Indeed,

$$
\begin{aligned}
& \left\langle R^{-1} r, b\right\rangle-\left\langle c, D^{-1} A^{T} R^{-1} b\right\rangle \\
& =\left\langle R^{-1} r, b\right\rangle-\left\langle\left(D^{-1} A^{T} R^{-1}\right)^{T} c, b\right\rangle \\
& =\left\langle R^{-1} r, b\right\rangle-\left\langle R^{-1} A D^{-1} c, b\right\rangle=0, \\
& -\left\langle D^{-1} c, c\right\rangle+\left\langle c, D^{-1} A^{T} R^{-1} r\right\rangle+\left\langle R^{-1} r, r\right\rangle-\left\langle R^{-1} r, r\right\rangle \\
& =-\left\langle D^{-1} c, c\right\rangle+\left\langle c, D^{-1} A^{T} R^{-1} r\right\rangle+\left\langle A^{T} R^{-1} r, D^{-1} c\right\rangle \\
& -\left\langle A^{T} R^{-1} r, D^{-1} A^{T} R^{-1} r\right\rangle \\
& =-\left\langle c-A^{T} R^{-1} r, D^{-1}\left(c-A^{T} R^{-1} r\right)\right\rangle\langle 0 .
\end{aligned}
$$

In the last expression, the inequality is strict, because by virtue of the conditions of Theorem $1\left(c-A^{T} R^{-1} r\right) \neq 0$. Besides, $d>d_{0}=\left\langle R^{-1} b, b\right\rangle$.

Then from (4) taking into account that $\lambda^{0}>0$ (the negative value of $\lambda^{0}$ corresponds to the minimum in the problem (1)), we obtain

$$
\begin{aligned}
& \lambda^{0}=\frac{1}{2} \sqrt{\frac{\left\langle D^{-1} c, c\right\rangle-\left\langle c, D^{-1} A^{T} R^{-1} r\right\rangle}{d-\left\langle R^{-1} b, b\right\rangle}} \\
&=\frac{1}{2} \sqrt{\frac{\left\langle D^{-1} c, c-A^{T} R^{-1} r\right\rangle}{d-\left\langle R^{-1} b, b\right\rangle}}, \\
& \mu^{0}=R^{-1}\left(r-2 \lambda^{0} b\right), \quad x^{0}=\frac{1}{2 \lambda^{0}} D^{-1}\left(c-A^{T} \mu^{0}\right) .
\end{aligned}
$$

Substituting the expressions for the matrix $R$ and the vector $r$, we obtain formulas (2).

We have obtained in explicit form a solution of the problem of maximizing a linear function with linear constraints of the type of equations and a quadratic constraint of the type of inequality. Naturally, the question arises: what will happen in the case of linear inequality constraints, in particular, when the condition of non-negativity of $x$ is present. In this case, KKT-conditions give us the system of linear equations for non-zero components of the vectors $x^{0}$ and active constraints. Therefore, in all the formulas obtained the "reduced" vectors will appear, and there will be instead of the matrices $A$ and $D$ some of their sub-matrices (square for $D$ ). Of course, this will lead to a back-to-back algorithm and complicate calculations. However, for not very large dimensions everything is quite computable.

Here we consider those changes in the solution that are associated with the addition of the non-negativity condition $x \geq 0$, which is widespread in the LP. In this case,

$$
d \geq \tilde{d}_{0}=\min _{x \in \tilde{X}_{0}}\langle D x, x\rangle, \quad \tilde{X}_{0}=\{x \mid A x=b, \quad x \geq 0\},
$$


KKT-conditions for the problem (1) have the form:

$$
\begin{gathered}
\frac{\partial L\left(x^{0}, \lambda^{0}, \mu^{0}\right)}{\partial x_{i}}=0, x_{i}^{0}>0, \\
\frac{\partial L\left(x^{0}, \lambda^{0}, \mu^{0}\right)}{\partial x_{i}} \leq 0, x_{i}^{0}=0, i=1, \ldots, n .
\end{gathered}
$$

For nonzero components $x^{0}$ we have the system of equations: $\tilde{c}-2 \lambda \tilde{D} x^{0}-\tilde{A}^{T} \mu=0$, where $\tilde{D}$ is a square sub-matrix of the matrix $D$ obtained by deleting rows and columns with numbers corresponding to the zero components of $x^{0}, \tilde{A}$ is a sub-matrix of the matrix $A$ with deleted corresponding rows, $\tilde{x}^{0}$ is the vector of nonzero components $x^{0}, \tilde{c}$ is the vector of corresponding coefficients. Square sub-matrices of a positive definite matrix $D$ are also positive definite and, consequently, non-degenerate. In this case $\lambda \neq 0$, if the condition that the vector $c$ does not belong to the subspace of vector-rows of the matrix $A$ is replaced by a stronger condition for all sub-vectors. Therefore, we have

$$
\tilde{\chi}^{0}=\frac{1}{2 \lambda^{0}} \tilde{D}^{-1}\left(\tilde{c}-\tilde{A}^{T} \mu^{0}\right),
$$

and in the expressions for $\lambda^{0}$ and $\mu^{0}$ there are also submatrices $\tilde{D}$ and $\tilde{A}$. For the given case, KKT-conditions are necessary and sufficient, so the search stops as soon as the point satisfying them is found.

We note, that from the point of view of our problem solution, the condition that quadratic restriction is active is not so significant, because if it is not active and $\lambda=0$, then we simply obtain the LP problem as an alternative.

It is possible to reduce the search using the idea of the branch and bound algorithm. The formulas obtained on the basis of the KKT conditions allow us to find simultaneously the minimum of the linear criterion (the second negative solution of the quadratic equation (4)). Therefore, the solutions found for the initial matrix, if they do not satisfy the condition of non-negativity, give us the upper and lower bounds for the desired maximum. For the submatrices of lower dimensions considered further, the solutions found, if they do not satisfy the condition of nonnegativity, give new lower and upper bounds. If for some sub-matrix the upper bound turns out to be less than the general current lower one, then its sub-matrices are not considered (since consideration of the sub-matrix means assignment of zero values to a part of the components of the vector $x$, then the maximum can only decrease).

The LP problem with inequality-type constraints can be reduced to the LP problem with equality-type constraints by introducing non-negative slack variables, i.e. formally to the case considered above. However, it is necessary to clarify the conditions for the existence of the so- lution. Therefore, this issue requires a separate consideration.

\section{Correction of the right-hand side of the constraints of the improper linear programming problem}

We consider the LP problem with inconsistent constraints of the following type:

$$
\langle c, x\rangle \rightarrow \max _{x \in X}, X=\left\{x \mid A_{1} x=b_{1}, A_{2} x=b_{2}\right\}=\emptyset .
$$

In the constraints of the problem (5) it is assumed that the system of equations $A_{1} x=b_{1}$ is consistent and not subject to correction (so-called, directive restrictions), and the equations $A_{2} X=b_{2}$ are inconsistent and we can correct its right-hand side. The value of correction of the right-hand side in the Euclidean norm is $\left\langle A_{2} x-b_{2}, A_{2} x-b_{2}\right\rangle$.

The division into two types of restrictions (directive and optional) is rather arbitrary. In fact, this is one incompatible system of constraints, but the correction task is complicated by a ban on correcting part entries of the vector of the right-hand side of the constraints. This problem is embedded in the previous problem of linear-quadratic programming, and therefore its solution is obtained as a corollary (provided that the corrected part of the equations is over-determined).

As we noted above, it was proposed in [5] to consider the correction of the improper LP problem as a two-criteria problem, which is formalized in the form of minimization of the spectral norm or the Frobenius norm of the correction matrix under the restriction from below to the initial linear criterion. Here we consider in some sense the inverse problem, namely, the problem of maximizing the initial criterion in (5) under the restriction from above to the Euclidean norm of correction (or discrepancy) of the vector of the right-hand side of the constraints.

To formalize this problem in the form of a special case of the problem (1), we introduce augmented vectors $\bar{c}=$ $(0, c)^{T}, z=(1, x)^{T}, e=(1,0, \ldots, 0)^{T}$ and matrixes $\bar{A}=$ $\left(\begin{array}{cc}1 & 0 \ldots 0 \\ -b_{1} & A_{1}\end{array}\right), B=\left(\begin{array}{ll}-b_{2} & A_{2}\end{array}\right)$. Then the system of equations $A_{2} X=b_{2}$ is transformed into the homogeneous system $B z=0$, and the value of correction of the right-hand side of the system $A_{2} X=b_{2}$ is equal to $\langle B z, B z\rangle=\langle D z, z\rangle$, where $D=B^{T} B$ - symmetric non-negative definite matrix. As equations $A_{2} X=b_{2}$ are assumed to be inconsistent, the matrix $B$ has linearly independent columns, so $D$ is not degenerate. We introduce a restriction on the value of correc- 
tion $\langle D z, z\rangle \leq d$. The system $A_{1} x=b_{1}$ is transformed into $\bar{A} z=e$, taking into account the additional condition $z_{0}=1$ (in the augmented vector $z$ the first component is $z_{0}$ ). We obtain the problem of the form (1)

$$
\langle\bar{c}, z\rangle \rightarrow \max _{z \in Z}, Z=\{z \mid\langle D z, z\rangle \leq d, \bar{A} z=e\} .
$$

Corollary of the Theorem 1. If the system of equations $\bar{A} z=e$ is consistent, the matrix $B$ has linearly independent columns, $d>\left\langle\left(\bar{A} D^{-1} \bar{A}^{T}\right)^{-1} e, e\right\rangle$, the vector $\bar{c}$ does not belong to the subspace of vector-rows of the matrix $\bar{A}$, then the problem (6) has a solution and it can be represented in the form

$$
\begin{aligned}
& z^{0}=\frac{1}{2 \lambda^{0}} D^{-1}\left(\bar{c}-\bar{A}^{T} \mu^{0}\right), \\
& \lambda^{0}=\frac{1}{2} \sqrt{\frac{\left\langle D^{-1} \bar{c}, \bar{c}-\bar{A}^{T}\left(\bar{A} D^{-1} \bar{A}^{T}\right)^{-1}\left(\bar{A} D^{-1} \bar{C}\right)\right\rangle}{d-\left\langle\left(\bar{A} D^{-1} \bar{A}^{T}\right)^{-1} e, e\right\rangle},} \\
& \mu^{0}=\left(\bar{A} D^{-1} \bar{A}^{T}\right)^{-1}\left(\bar{A} D^{-1} \bar{c}-2 \lambda^{0} e\right),
\end{aligned}
$$

and the optimal solution of the problem (5) $x^{0}$ is obtained from $z^{0}$ by excluding the first component $z_{0}^{0}=1$.

If there are no directive constraints in the problem (5), then we have $e=1, \bar{A}=(1,0, \ldots, 0)$. If in the problem (5) there are non-negativity conditions $x \geq 0$, then in all the above formulas "reduced" vectors and sub-matrices will appear (see Section 2).

Example 1. Consider the problem

$$
\begin{aligned}
x_{1} & \rightarrow \max _{x \in X}, X \\
0 \cdot x_{1} & \left.=\sqrt{2}, 1 \cdot x_{2}=0, x_{1}+x_{2}=2\right\}=\emptyset .
\end{aligned}
$$$$
=\left\{x \mid x_{1}+x_{2}=1\right. \text { with an inconsistent system of constraints, is }
$$

Here $c=(1,0)^{T}$, one directive constraint $x_{1}+x_{2}=1$, i.e. $A_{1}=(1,1)$ and $b_{1}=1$, three constraints are correctable, i.e.

$$
\begin{aligned}
A_{2} & =\left(\begin{array}{ll}
0 & 0 \\
0 & 1 \\
1 & 1
\end{array}\right), \quad b_{2}=\left(\begin{array}{c}
\sqrt{2} \\
0 \\
2
\end{array}\right), \\
\bar{c} & =\left(\begin{array}{l}
0 \\
1 \\
0
\end{array}\right), \quad B=\left(\begin{array}{ccc}
-\sqrt{2} & 0 & 0 \\
0 & 0 & 1 \\
-2 & 1 & 1
\end{array}\right), \\
D & =\left(\begin{array}{ccc}
6 & -2 & -2 \\
-2 & 1 & 1 \\
-2 & 1 & 2
\end{array}\right), \quad \bar{A}=\left(\begin{array}{ccc}
1 & 0 & 0 \\
-1 & 1 & 1
\end{array}\right), \\
e & =\left(\begin{array}{l}
1 \\
0
\end{array}\right) .
\end{aligned}
$$

Solution of the problem (3) gives us $d_{0}=3$ (and $\tilde{d}_{0}=3$ also).
Let $d=4$, then $r=(1,2)^{T}, R=\left(\begin{array}{ll}0.5 & 0.5 \\ 0.5 & 1.5\end{array}\right), \lambda^{0}=0.5$, $\mu^{0}=(-2,2)^{T}, z^{0}=(1,2,-1)^{T}, x^{0}=(2,-1)^{T}$. The right-hand side vector of the constraints after correction is $(0,-1,1)^{T}, \Delta b_{2}=A_{2} x^{0}-b_{2}=(-\sqrt{2},-1,-1)^{T}$.

If we add the condition $x \geq 0$ in this example, then for $d=4$ the constraint $\langle D z, z\rangle \leq d$ in (6) is not active, and $\lambda^{0}=0$. We have LP problem, which solution is $x^{0}=(1,0)^{T}$; $\Delta b_{2}=A_{2} x^{0}-b_{2}=(-\sqrt{2}, 0,-1)^{T}$.

\section{Correction of both sides of the constraints of the improper linear programming problem}

We now consider the more complicated problem of maximizing the initial criterion for an improper LP problem with a restriction from above to the value of the Frobenius norm of the correction matrix of both sides of equalitytype constraints (all data are corrected). This problem will be reduced also to the form (1) and we use the developed method for its solution. The difference is that it is already not convex (with at least one negative eigenvalue, so in general, NP-hard [9]). Therefore, the extremum conditions in the general case are only necessary, which, in the presence of the condition of non-negativity, leads to the necessity of enumeration of all sub-matrices.

The initial improper LP problem, namely the problem

$$
\langle c, x\rangle \rightarrow \max _{x \in X}, X=\{x \mid A x=b\}=\emptyset .
$$

To formalize the problem (7) as a particular case of the problem (1), we introduce augmented vectors $\bar{c}=\left(c_{0}, c\right)^{T}$, $z=(1, x)^{T}$ and matrices $\bar{H}=(-h H), B=(-b A)$. Then the corrected system of equations, under which $X \neq \emptyset$, is $B z=$ $-\bar{H} z$. It is known that the minimum of the square norm of the correction matrix for fixed $z$ is $\min \|\bar{H}\|^{2}=\frac{\|B z\|^{2}}{\|z\|^{2}}$ and $\bar{H}=\frac{\left[-B z, z^{T}\right]}{\langle z, z\rangle}$ (here in square brackets is the product of vectors as matrices, vectors norm is Euclidean, matrixes norm is Frobenius, which is equal to Euclidean norm if conceding it as a vector). Since in this case the rank of the matrix $\bar{H}$ is equal to 1 , its spectral norm is equal to the Frobenius norm. It is required to find $z$ such that the objective function reaches its maximum value under the condition that the square norm of the correction matrix does not exceed some $\varepsilon$ : $\frac{\| B z||^{2}}{\|z \mid\|^{2}} \leq \varepsilon$. The numerator of the left-hand side of the system of inequality is $\langle B z, B z\rangle=\langle D z, z\rangle$, where $D=B^{T} B$ - symmetric non-negative definite matrix. Then the prob- 
lem with limited correction is

$$
\langle\bar{c}, z\rangle \rightarrow \max _{z \in Z}, \quad Z=\left\{z \mid\langle D z, z\rangle \leq \varepsilon\langle z, z\rangle, z_{0}=1\right\} .
$$

The quadratic constraint in (8) can be rewritten as $\langle(D-\varepsilon I) z, z\rangle \leq 0$, where $I$ is the identity matrix, so (8) looks like (1). But there is the difference, because $(D-\varepsilon I)$ is not positive definite matrix. Since $z_{0}=1, c_{0}$ in the problem (8), in general, can be arbitrary, in particular, zero, but it turns out that the method of solution considered below requires the selection of its certain value.

The infimum of square norm (Frobenius or spectral) of the correction matrix for which $X \neq \emptyset$ equals $\mu_{1}(D)-$ the first (minimal) eigenvalue of the matrix $D$, and minimal correction matrix exists if and only if corresponding eigenvectors have a non zero first component [5]. If $\operatorname{rank} B<n(n$ -number of its columns), than $\mu_{1}(D)=0$ and the problem of minimal correction has no solution (the system of equations $A x=b$ becomes consistent under arbitrary small correction, but its solution has arbitrarily large norm). So the considered problem of minimal correction has sense, if $\operatorname{rank} B=n$ and the system of equations $A x=b$ is overdetermined. It is known, that $\mu_{1}(D) \leq \mu_{1}\left(A^{T} A\right) \leq \mu_{2}(D)$ ), where $\mu_{2}(D)$ is the second eigenvalue of the matrix $D$. If $\mu_{1}(D)<\mu_{1}\left(A^{T} A\right)$, then minimal correction matrix exists and is unique [10] (otherwise again solutions of corrected system of equations have arbitrarily large norm). Therefore, we will assume further that $\mu_{1}(D)<\mu_{1}\left(A^{T} A\right)$. This condition is sufficient for the rank of matrix $B$ to be equal to the number of its columns.

Theorem 2. If $\mu_{1}(D)<\mu_{1}\left(A^{T} A\right)$ and the parameter $\varepsilon$ belongs to the interval $\left(\mu_{1}(D), \mu_{1}\left(A^{T} A\right)\right)$, the vector $\bar{c}=$ $\left(c_{0}, c\right)^{T}, c_{0}$ is the minor root of the quadratic equation

$$
k_{00} c_{0}^{2}+2\left(\sum_{j=1}^{n} k_{0 j} c_{j}\right) c_{0}+\sum_{i, j=1}^{n} k_{i j} c_{i} c_{j}=0,
$$

where $k_{i j}$ are entries of the matrix $(D-\varepsilon I)^{-1}$, the first component $a_{0}$ of the $(n+1)$-dimensional vector $(D-\varepsilon I)^{-1} \bar{c}$ is not equal to zero, then the problem (8) has a solution and it can be represented in the form $z^{0}=\frac{1}{a_{0}}(D-\varepsilon I)^{-1} \bar{c}$, the correction matrix is $\bar{H}=\frac{\left[-B z^{0}, z^{0 T}\right]}{\left\langle z^{0}, z^{0}\right\rangle}$, the solution of the corrected system of equations $x^{0}$ is obtained from $z_{0}$ by excluding the first component $z_{0}^{0}=1$.

Proof. Because $\mu_{1}(D)<\varepsilon<\mu_{2}(D), \varepsilon$ does not belong to the spectrum of $D$, the matrix $(D-\varepsilon I)$ is non-degenerate. The inequality $\mu_{1}(D)<\mu_{1}\left(A^{T} A\right)$ is a sufficient condition for existents of a minimal correction matrix. A minimal correction matrix exists if and only if an eigenvector corresponding $\mu_{1}(D)$ have a non zero first component. So such eigenvector exists, and its first component may be considered equal to 1 . Obviously, it belongs to the set $Z$, so $Z \neq \emptyset$. Besides, the set $Z$ is bounded. Indeed, suppose the contrary. As $z_{0}=1$, we have the following equality $\langle D z, z\rangle=-\langle b, b\rangle+\left\langle\left(A^{T} A\right) x, x\right\rangle$. We represent $x$ in the form $x=\alpha \bar{e},\|\bar{e}\|=1$. Then the restriction in (8) has the form $-\langle b, b\rangle+\alpha^{2}\left\langle\left(A^{T} A\right) e, e\right\rangle \leq \varepsilon\left(1+\alpha^{2}\right)$. For $\alpha \rightarrow \infty$ we have $\left\langle\left(A^{T} A\right) e, e\right\rangle \leq \varepsilon<\mu_{1}\left(A^{T} A\right)$ and obtain a contradiction. Since a linear function reaches an extreme value on the boundary of the set $Z$, the constraint in (8) is satisfied as equality at the optimal point. The Lagrange function for the problem (8) is $L(x, \lambda)=\langle\bar{c}, z\rangle+\frac{1}{2} \lambda(\varepsilon\langle z, z\rangle-\langle D z, z\rangle)$. The necessary conditions of maximum lead to the system of equations: $\bar{c}+\lambda(\varepsilon z-D z)=0$, whence $\lambda^{0} \neq 0$ and $z^{0}=\frac{1}{\lambda^{0}}(D-\varepsilon I)^{-1} \bar{c}$. From the necessary conditions for the optimal $z^{0}$ we have $\left\langle\bar{c}, z^{0}\right\rangle+\lambda^{0}\left(\varepsilon\left\langle z^{0}, z^{0}\right\rangle-\left\langle D z^{0}, z^{0}\right\rangle\right)=0$, so $\left\langle\bar{c}, z^{0}\right\rangle=0$. Taking into account, that $z_{0}^{0}=1$, we get $\left\langle\bar{c}, x^{0}\right\rangle=-c_{0}$. So the maximum value of the objective function in the problem (7) after correction is equal to $-c_{0}$. We substitute $z^{0}$ into $\left\langle\bar{c}, z^{0}\right\rangle=0$ : $\left\langle\bar{c}, \frac{1}{\lambda^{0}}(D-\varepsilon I)^{-1} \bar{c}\right\rangle=0$ or $\left\langle\bar{c},(D-\varepsilon I)^{-1} \bar{c}\right\rangle=\sum_{i, j=0}^{n} k_{i j} c_{i} c_{j}=0$, where $k_{i j}$ are entries of the matrix $(D-\varepsilon I)^{-1}$. We obtain the quadratic equation to find $c_{0}: \alpha c_{0}^{2}+\beta c_{0}+\gamma=0$, where $\alpha=k_{00}, \beta=$ $2 \sum_{j=1}^{n} k_{0 j} c_{j}, \gamma=\sum_{i, j=1}^{n} k_{i j} c_{i} c_{j}$. Because our problem has a solution under selected $\varepsilon$, this equation has real roots and we must choose a minor one. Then we substitute $\bar{c}$ in $z^{0}=\frac{1}{\lambda^{0}}(D-\varepsilon I)^{-1} \bar{c}$ and taking into account that $z_{0}^{0}=1$, we obtain that $\lambda^{0}$ is the first component of the vector $(D-\varepsilon I)^{-1} \bar{c}$, $x^{0}$ is obtained from $z^{0}$ by excluding the first component, the correction matrix is $\bar{H}=\frac{\left[-B z^{0} z^{0 T}\right]}{\left\langle z^{0} z^{0}\right\rangle}$.

If in the problem (7) there are non-negativity conditions $x \geq 0$, then in all the above formulas "reduced" vectors and sub-matrices will appear. In this case the minimum of square norm of the correction matrix for which $X \neq \emptyset$ equals to the minimum of eigenvalues of the matrix $D$ and sub-matrixes $\tilde{D}$ for which there exist nonnegative eigenvectors [5]. The admissible $\varepsilon$ must be greater than this value.

Example 2. Consider the problem

$$
\begin{aligned}
x_{1}+x_{2} & \rightarrow \max _{x \in X}, \\
X & =\left\{x \mid 2 x_{1}+x_{2}=0, x_{1}+2 x_{2}=0,\right. \\
& \left.-x_{1}+x_{2}=0, x_{1}-x_{2}=1\right\}=\emptyset .
\end{aligned}
$$




$$
\begin{aligned}
& \begin{array}{l}
\text { Here } A=\left(\begin{array}{cc}
2 & 1 \\
1 & 2 \\
-1 & 1 \\
1 & -1
\end{array}\right), b= \\
\left.0 \begin{array}{ccc}
0 & 2 & 1 \\
0 & 1 & 2 \\
0 & -1 & 1 \\
-1 & 1 & -1
\end{array}\right), D=\left(\begin{array}{ccc}
1 & -1 & 1 \\
-1 & 7 & 2 \\
1 & 2 & 7
\end{array}\right), \\
(D) \approx 0.551 \text { (corresponding eigenvector } \\
\left.z^{*}=(1,0.225,-0.225)^{T},\left\langle\bar{c}, x^{\star}\right\rangle=0\right) \\
\left(A^{T} A\right)=5, \mu_{2}(D) \approx 5.449 . \\
\text { We impose a restriction on the correction } \\
\text { the constraints with } \varepsilon=1, i . e .\|\bar{H}\|^{2} \leq 1 . \\
(D-\varepsilon I)=\left(\begin{array}{ccc}
0 & -1 & 1 \\
-1 & 6 & 2 \\
1 & 2 & 6
\end{array}\right),
\end{array} \\
& (D-\varepsilon I)^{-1}=\left(\begin{array}{ccc}
-2 & -\frac{1}{2} & \frac{1}{2} \\
-\frac{1}{2} & \frac{1}{16} & \frac{1}{16} \\
\frac{1}{2} & \frac{1}{16} & \frac{1}{16}
\end{array}\right) \text {. }
\end{aligned}
$$

The quadratic equation for finding $c_{0}$ is $2 c_{0}^{2}-\frac{1}{4}=0$, whence $c_{0}= \pm 0.25 \sqrt{2}$. The objective function $\left\langle\bar{c}, x^{0}\right\rangle=$ $-c_{0}$ takes the maximum value when $c_{0}=-0.25 \sqrt{2} \approx$ -0.354 . Then $\bar{c}=\left(\begin{array}{c}-0.354 \\ 1 \\ 1\end{array}\right), z^{0}=\frac{1}{\lambda^{0}}(D-\varepsilon I)^{-1} \bar{c}=$ $\frac{1}{\lambda^{0}}\left(\begin{array}{c}0.707 \\ 0.302 \\ -0.052\end{array}\right)$. Taking into account that $z_{0}^{0}=1$, we have $\lambda^{0}=$ $0.707, z^{0}=\left(\begin{array}{c}1 \\ 0.427 \\ -0.073\end{array}\right), x^{0}=\left(\begin{array}{c}0.427 \\ -0.073\end{array}\right)$.

In this case the correction matrix

$$
\bar{H}=\frac{\left[-B z^{0}, z^{0^{T}}\right]}{\left\langle z^{0}, z^{0}\right\rangle}=\left(\begin{array}{ccc}
-0.657 & -0.28 & 0.048 \\
-0.236 & -0.101 & 0.017 \\
0.421 & 0.18 & -0.031 \\
0.421 & 0.18 & -0.031
\end{array}\right) \text {. }
$$

If we add the condition $x \geq 0$ in this example, the minimum of square norm of the correction matrix for which $X \neq \emptyset$ equals to the minimum eigenvalue of the sub-matrix $\tilde{D}=\left(\begin{array}{cc}1 & -1 \\ -1 & 7\end{array}\right)$, which is 0.838 (corresponding eigenvector $(0.987,0.16)^{T}$ is nonnegative). So we can left $\varepsilon=1$. In this case the solution corresponds to sub-matrix $\tilde{D}$. Then $(\tilde{D}-\varepsilon \tilde{I})^{-1}=\left(\begin{array}{cc}-6 & -1 \\ -1 & 0\end{array}\right)$, the quadratic equation for finding $c_{0}$ is $-6 c_{0}^{2}-2 c_{0}=0$, whence $c_{0}=0$ or $-\frac{1}{3}$. The objective function $\left\langle\bar{c}, x^{0}\right\rangle=-c_{0}$ takes the maximum value when $c_{0} \approx-0.333$. Then $z^{0}=\left(\begin{array}{c}1 \\ 0.333 \\ 0\end{array}\right), x^{0}=\left(\begin{array}{c}0.333 \\ 0\end{array}\right)$, the correction matrix

$$
\bar{H}=\frac{\left[-B z^{0}, z^{0^{T}}\right]}{\left\langle z^{0}, z^{0}\right\rangle}=\left(\begin{array}{ccc}
-0.6 & -0.2 & 0 \\
-0.3 & -0.1 & 0 \\
0.3 & 0.1 & 0 \\
0.6 & 0.2 & 0
\end{array}\right) .
$$

\section{Conclusion}

Thus, we have obtained a solution to the problem of maximizing a linear function with linear constraints of the equality type and a quadratic restriction of the inequality type. We note that problems of this kind have been studied in a number of papers (for example, $[11,12]$ ) and they offer iterative solution algorithms. The method proposed by us allows to apply standard matrix calculations and has been verified in a number of comparatively simple examples (two of them are given in the article). However, it is too early to speak about the comparative effectiveness of numerical methods. The main content of the work is its application to the new problems of the limited correction of the improper LP problem. However, the scope of applications of the results obtained is much broader. In the future, we plan to apply them to more general problems of correction of improper and unstable problems of linear and quadratic programming. In particular, we have obtained the results of the problem of limited correction of the left-hand side of the restrictions of the improper LP problem (the vector $b$ is fixed). This task has a certain specificity, concerning the issues of solution existence and the method of its searching. The corresponding results are published in [8].

We plan also to apply the results of this paper in regression analysis (smoothing of the piecewise linear regression, the condition of two samples trends proximity, etc.). We note that in these problems the over-determination of the system of equations and the absence of the condition of non-negativity are automatically satisfied.

\section{References}

[1] Alizadeh F., Interior point methods in semidenite programming with applications to combinatorial optimization, SIAM Journal on Optimization, 1995, 1(5), 13-51

[2] Golub G.N., Van Loan C.F., An analysis of the total least squares problems, SIAM J. Numer. Anal. 1980, 17, 883-893 
[3] Van Huffel S., Vandewalle J., Algebraic relationships between clas sical regression and total least-squares estimation, Linear Algebra and its Applications, 1987, 93, 149-160

[4] Eremin I.I., Mazurov V.D., Astaf'ev N.N., Improper linear and convex programming problems, Nauka, Moscow, 1983

[5] Gorelik V.A., Matrix correction of a linear programming problem with inconsistent constraints, Computational Mathematics and Mathematical Physics, 2001, 11(41), 1615-1622

[6] Erohin V.I., Matrix correction of a dual pair of improper linear programming problems, Computational Mathematics and Mathematical Physics, 2007, 47(4), 564-578

[7] Gorelik V.A., Zolotova T.V., The problem of linear-quadratic programming. In: Proceedings of 8th International Conference on Optimization and Applications (2-7 October 2017, Petrovac, Montenegro), CEUR-WS, 2017, 1987, 233-238
[8] Gorelik V.A., Zolotova T.V., Approximation of the improper linear programming problem with restriction on the norm of the correction matrix of the left-hand side of the constraints, In: Proceedings of 9th International Conference on Optimization and Applications, (1-5 October 2018, Petrovac, Montenegro), DEStech Transactions on Computer Science and Engineering, 2018, 14-24

[9] Pardalos P., Vavasis S., Quadratic programming with one negative eigenvalue is NP-hard, Journal of Global Optimization, 1991, 1(1), 15-22

[10] Gorelik V.A., Erohin V.I., Optimal matrix correction of inconsistent systems of linear algebraic equations by minimal Euclidean norm, CC RAS, Moscow, 2004

[11] Floudas C.A., Pardalos P.M. (Editors), Encyclopedia of Optimization, Springer, Boston, MA, 2009

[12] Martein L., Schaible S., On solving a linear program with one quadratic constraint, Decisions in Economics and Finance, 1987, 10(1-2), 75-90 